\title{
Aplicación del modelo gravitatorio al estudio de las migraciones internas. El caso de las comunas en las Regiones de Los Ríos y Los Lagos, Chile entre los años 1977-2012. \\ Application of the gravity model to the study of Internal Migrations. The case of the communes in the Los Ríos and Los Lagos Chile, between 1977-2012 years.
}

\author{
Francisco Maturana Miranda, Alonso Jara Jara
}

\begin{abstract}
Resumen
La aplicación del modelo gravitatorio al estudio de flujos posee una extensa tradición en la ciencia geográfica. Este trabajo analiza la base teórica que fundamenta el modelo gravitatorio circunscrito en el concepto de interacción espacial. Posteriormente, se testea dicho modelo aplicándolo al estudio de las migraciones internas en comunas de las Regiones de los Lagos y Ríos en los periodos censales comprendidos entre 1977 y 2012. Se planteó la hipótesis que las estructuras migratorias ha sido más bien estables y circunscritas en los diferentes espacios regionales. Así, se demuestra la fiabilidad del modelo, la caracterización de efectos barreras y las relaciones preferenciales en el territorio. Finalmente, se concluye un patrón espacial que tiende a la concentración de los flujos en las capitales regionales y donde las migraciones en los últimos 20 años están circunscritas a cada espacio regional pese que la Región de Los Ríos perteneció a la de Los Lagos.
\end{abstract}

Palabras clave: Modelo gravitatorio, Migración interna, Región de Los Ríos, Región de Los Lagos.

\begin{abstract}
The use of the gravitational model in the study of flows has a long tradition in the geographic science. This paper analyzes the theoretical basis of the gravitational model based on the concept of spatial interaction. Subsequently, this model is tested by applying it to the study of internal migration between communes of the Los Lagos and Los Ríos Regions in the census periods between 1977 and 2012. It was assumed that migration structures have been rather stable and circumscribed in the different regional spaces. Thus, the reliability of the model, the characterization of barrier effects and the preferential relations in the territory are demonstrated. Finally, a spatial pattern is stablished that focuses on the concentration of flows in regional capitals and where migrations in the last 20 years have been limited to each regional space despite the Los Rios Region belonged to the Los Lagos Region.
\end{abstract}

Keywords: Gravity model, Internal migration, Los Ríos Region, Los Lagos Region.

Recibido el 18 de enero de 2018, aceptado el 23 de marzo de 2018.

Departamento de Geografía, Universidad Alberto Hurtado. Cienfuegos 41, Santiago, Chile.

E-mail: fmaturana@uahurtado.cl

Este artículo se inserta en el Proyecto Fondecyt Iniciación No.11150087 


\section{Introducción}

El territorio es una producción resultante de la interacción de distintos elementos en el espacio, donde los flujos expresan relaciones de atracción-rechazo y las representaciones de dichos fenómenos o potenciales fenómenos (Pumain \& Saint-Julien, 2001; Levy \& Lussault, 2013; Batty, 2013).

Si bien no existe una delimitación unánime del concepto de interacción espacial, debido principalmente a la forma de medición, interpretación y justificación de los diversos procesos que producen (Parrochia, 2006). Pumain \& Saint-Julien (2001); Batty (2013); Maturana, Rojas, \& Poblete (2016) señalan a la interacción espacial como un pilar fundamental en el análisis espacial y por tanto de la ciencia geográfica.

Pero ¿cómo un concepto difuso y difícil de delimitar puede ser catalogado como núcleo central de la geografía? Según Pumain \& Saint-Julien (2001) del concepto de interacción espacial se desprende la producción social del espacio geográfico, en el cual los actores y/o elementos de este interactúan entre sí, generando relaciones de acciónreacción determinadas por posiciones, jerarquizaciones y roles de aquellos. Es decir, sin la interacción espacial el espacio geográfico carecería de movimiento y existirían solamente individuos aislados, puesto que tal interacción es construida en base a desplazamientos, intercambios y comunicaciones entre estos.

Por ende, la interacción espacial se podría caracterizar como el flujo resultante del movimiento a escala espacio temporal de mediano o largo plazo de elementos, los cuales poseen un punto de origen y uno de destino, ambos con una localización (Batty, 2013).
Comúnmente se caracteriza el flujo como una medida agregada con cierta regularidad o patrón, es decir es una superposición de movilidades particulares e individuales de los diversos elementos espaciales (personas, información, bienes, ideas, tráfico, visitas a páginas web, llamadas de celular, etc.) que se desenvuelven por algún medio de transporte o comunicación (canal) a una escala temporal determinada (Pumain \& Saint-Julien, 2001; Batty, 2013).

Bajo tal contexto, los flujos y la interacción espacial han sido estudiados a partir de diversos modelos que buscan predecir la dirección, tamaños, intensidad de éstos y las configuraciones espaciales resultantes de tales procesos (Pumain \& Saint-Julien, 2001). En tal línea argumentativa, dos modelos son posibles de destacar. El primero, corresponde al modelo gravitatorio, uno de los más empleados y con una vasta tradición en el estudio y análisis en geografía (Commenges, 2016; Thomas \& Hugget, 1980; Parrochia, 2006). Un segundo tipo, corresponde a los de radiación, cuyos aportes han sido más bien recientes y se transforman en una alternativa para para el estudio de la interacción espacial (Stefanouli \& Polyzosa, 2017), haciendo hincapié en las problemáticas de los primeros en términos de la robustez de la estimación de sus parámetros, requerimiento de flujos, tratarse de un modelo determinista, entre otras (Kang, Liu, Guo, \& Qin, 2015; Simini, González, Maritan, \& Barabási, 2012).

Respecto al primero -gravitatorio- dicho modelo se basa en los aportes de Isaac Newton respecto a la ley de gravedad, aunque en este caso, se adaptó a las ciencias sociales. En tal área del conocimiento, no existe una única versión del modelo gravitatorio, puesestesehaidoconstruyendo en el tiempo a partir de diferentes aportes. Así, las primeras aproximaciones eran más 
bien intuitivas y vinculadas a la migración, como se observa en los pioneros trabajos de Ravenstein $(1885,1889)$ los cuales caracterizaban las migraciones con las nociones de población, entradas y salidas para un vasto conjunto de países europeos.

Hacia los años 1930 W.J. Reilly, plantea su modelo gravitatorio del comercio que daba un paso relevante en lo que vendría posteriormente. Tal aporte, consideraba las relaciones entre dos territorios y sus poblaciones, en el contexto de sus diferentes ventas. Posteriormente, fue la propuesta de Stewart $(1941,1948)$, que entrega un impulso para el despegue definitivo del modelo, presentando una formulación que se aproxima a lo que se verá en las siguientes propuestas. Esta se aprecia en la ecuación 1 que se representa a continuación:

$$
F i j=k P i P j / d i j(1)
$$

Donde el Fij representa el flujo total entre las zonas i y j, $\mathrm{k}$ es una constante determinada por el ajuste que se le quería entregar al modelo, Pi y $\mathrm{Pj}$ son las masas de cada zona o región (i y j respectivamente) y por último dij es la distancia que separa las dos zonas a estudiar.

Se tiene como premisa en la formulación de dicha ecuación lo siguiente, el flujo total estará vinculado proporcionalmente al producto de la masa total de los espacios y será inversamente proporcional a la distancia de dichos espacios, por ende, la multiplicación de las masas indica la probabilidad de interacción de elementos entre ambas zonas. Por último, destacar que la disminución de interacciones producto de una mayor distancia, produce un menor número de interacciones posibles, disminuyendo la probabilidad de interacción de los elementos espaciales (Ratti et al., 2010).

Posteriormente, desde la segunda mitad del siglo XX diversos autores realizan aportes que contribuyen a robustecer la metodología del modelo gravitatorio (Thomas \& Hugget, 1980; Haynes \& Fotheringham, 1984; Roy, 2004; Cheng \& Wang, 2008), entre ellos destaca Wilson (1971), el cual integra el principio maximización entropía al modelo permitiendo detectar el desplazamiento más probable de los flujos entre dos zonas (i y j), esto significa la especialización territorial de elementos que generan dichos flujos, la ecuación del modelo propuesto por Wilson es la siguiente:

$$
T i j=A i O i B j D j\left(e^{-\beta c i j}\right)(2)
$$

En donde Tij representa el flujo total entre dos zonas, $\mathrm{Ai}$ y $\mathrm{Bi}$ son parámetros de conservación entre flujos totales observados y estimados (doble restricción) permitiendo la misma cantidad de estos, Oi y Dj son las masas de las zonas y cij representa la distancia total entre $\mathrm{i}$ y $\mathrm{j}$. Un aspecto a destacar de este modelo es que, al poseer doble restricción y exponente negativo, asegura una correcta representación de los flujos y explicación de estos (Pumain \& Saint-Julien, 2001).

Con el transcurso de los años nuevos modelos más sofisticados han ido emergiendo, uno de ellos es el propuesto por Karamera, Iwuagwu, \& Davis (2000) en su estudio sobre los efectos de la migración internacional en América del Norte, donde los autores proponen que el flujomigratorio depende de los factores de oferta, demanda y otros restrictivos, planteando la siguiente ecuación:

$$
F i j t=A_{i j}^{a 0}\left(S_{i t}^{a 1} D_{j t}^{a 2} / R_{i j t}^{a 3}\right)
$$

Donde Fijt es el flujo total entre i y j, Sit factores de oferta, Djt factores de demanda, Rijtotras variablesaconsiderarquefavorecen o restringen la migración (temporalidad), Aij es una constante que contribuye a retener los flujos bilaterales ente i y j. 
En resumen, la utilidad de los modelos gravitatorios para el análisis de la interacción espacial radica en el análisis residual de valores obtenidos gracias a la resta resultante de los flujos reales menos los flujos modelados o estimados por el modelo. Así, tales valores residuales contribuirán a comprender los efectos barrera y preferencias de interacción entre diferentes espacios (Faura \& Gómez; 2001; Parrochia, 2006; Maturana et al., 2016).

Si bien el modelo posee una larga tradición de empleo en el estudio de interacciones, además de caracterizar óptimamente los flujos parcialmente homogéneos, tales como migración interurbana $o$ interregional, el modelo posee una baja robustez en cuanto respaldo teórico, incluso llegando a ser una de sus mayores debilidades, al tratarse de un modelo fijo que no toma en cuenta la temporalidad y transformación de la magnitud y dirección de los flujos (Duffus, Alfa, \& Soliman, 1987; Pumain \& Saint-Julien, 2001; Simini et al., 2012).

En cuanto a su aplicación, tal modelo ha sido desarrollado en diversas áreas de estudio como, por ejemplo: migración, comercio y economía, trafico, marketing, turismo, llamadas de teléfono, mediciones de partículas contaminantes, conmutación regional, movilidad, geopolítica, entre muchas otras aplicaciones (Pumain \& Saint-Julien, 2001; Chen \& Wang, 2008; Zubelzu, López, Gutiérrez, \& Blanco, 2011; Piras, 2016).

Una de las aplicaciones ya indicadas es el estudio de las migraciones, particularmente las de tipo interno. Éstas se pueden definir como un tipo de movilidad a escala subnacional y con una temporalidad que tiende a ser permanente o de larga duración, siendo uno de los fenómenos a nivel mundial más relevantes dado a que es parte central de los procesos de cambio demográfico de las naciones, además de estar intrínsecamente ligada a la ocupación del territorio, contribuyendo positivamente o negativamente al desarrollo de las entidades sub-nacionales (regiones, provincias y comunas en el caso chileno) (Busso, 2014).

Particularmente, se ha estudiado el impacto que presentan este tipo de procesos, considerando los vínculos que podrían ser atribuidos tanto a la oportunidad de trabajos como el impacto social en la construcción y evolución del territorio asociado al acto de migrar (Martínez, 2002; Rodrigo \& Atienza, 2014).

En este sentido, los procesos migratorios, estarían asociados a dos tipos, unos más bien de carácter voluntario y un segundo de tipo forzado (Eichenbaum, 1975). En estos últimos, existirán procesos donde la influencia de la sociedad, la independencia de éstas, determinaría los deseos que llevan al proceso de migrar. De esta forma y según lo planteando por Zelinsky (1971), tal migración se desarrollaría en diferentes fases que estarían vinculadas a los niveles de complejidad que presenta una sociedad. A su vez, tales procesos se podrían asociar a formas de movimiento internacional, domésticos (internos), rural-urbano, urbano-urbano (Ortiz \& Morales, 2002), determinados por una potencial migración según un determino nivel de circulación. Así, las escalas de la migración también serían diferenciadas, desde las de tipo campo-ciudad a pasar más bien a un proceso de movilidad en espacios metropolitanos (Zelinsky, 1971).

Si bien el fenómeno de las migraciones internas en Chile ha sido bien estudiado (Di Filippo \& Bravo, 1977; Schiappacasse, Contreras, \& Fuensalida, 2001; Instituto 
Nacional de Estadísticas (INE), 2012: 2017; Martínez, 2002; Rodríguez, 2004; Rodríguez \& González, 2006) no hay una gama de trabajos que aborden la temática migratoria interna desde la óptica metodológica del modelo gravitatorio y que consideren flujos más recientes. En este sentido, es posible, por ejemplo, destacar la propuesta de Maturana et al. (2016), en el cual se realiza una aplicación del modelo al estudio de las migraciones entre las comunas de la Región del Biobío. En tal trabajo se caracterizan los efectos barreras y preferenciales a partir de un perfil de los habitantes entre tales comunas. El tipo de modelo aplicado correspondió al tipo Wilson.
Es por esto y bajo la luz de lo expuesto, es que este artículo testeó la aplicación de una variación del modelo gravitatorio que caracterizó los flujos migratorios internos de las comunas de las Regiones de Los Ríos y Los Lagos (figura 1) con la finalidad de comprender los efectos barrera y preferenciales en la interacción migratoria de tales territorios.

Tales territorios, han presentado particularidades relevantes en los últimos años en la línea del auge de algunas ciudades intermedias del país (Maturana \& Rojas, 2015). En cuanto a sus características demográficas generales estas son posibles de observar en la tabla 1

Tabla 1

Antecedentes generales de las regiones en estudio. Fuente: Elaboración propia a partir de Barton \& Román (2016), INE (2017), Román, Barton, Bustos \& Salazar (2016), Vial, Maturana, \& Rojas (2016).

Table 1

General background in study regions. Source: Own elaboration based on Barton \& Román (2016), INE (2017), Román, Barton, Bustos, \& Salazar (2016), Vial, Maturana, \& Rojas (2016).

\begin{tabular}{llll}
\hline Región & Población & Concentración capital regional & Característica de relevancia \\
\hline Los Ríos & 384.837 & $43 \%$ en Valdivia & $\begin{array}{l}\text { Nueva región el año 2007 (antes } \\
\text { perteneciente a Los Lagos) } \\
\text { aumento considerable de la } \\
\text { inversión pública efectiva. }\end{array}$ \\
& 828.708 & $\begin{array}{l}\text { 35\% en Puerto Montt (con } \\
\text { Puerto Varas) }\end{array}$ & $\begin{array}{l}\text { Salmonera, catapultado a } \\
\text { tal región como el segundo } \\
\text { productor a nivel. }\end{array}$ \\
\hline
\end{tabular}

Así, este trabajo buscó explorar la presencia o no de cambios en los patrones migratorios antes y post la creación de la nueva región, que pudiesen ser relevantes de profundizar en otros estudios. En efecto, se observó la existencia o no de modificaciones en la estructura migratoria en diferentes periodos de tiempo, con la finalidad de analizar la incidencia o no de un límite político administrativo en este caso regional o incluso anterior al proceso desegmentación del espacio. Es decir, cuando el territorio de Los Ríos pertenecería al de Los Lagos, en ese caso límite provincial. Si bien el periodo seleccionado no es de larga data, de todos modos, resulta relevante analizar tal proceso, como un proxi a estudios futuros que podrían profundizar en lo planteado.

Así el objetivo de este trabajo estuvo orientado a caracterizar los flujos 
migratorios entre las comunas de las dos regiones ya indicadas mediante el modelo gravitatorio de la tal forma de identificar y comprender patrones espaciales en el comportamiento de tal interacción antes y post el proceso de regionalización, planteado la hipótesis que las estructuras migratorias ha sido más bien estables y circunscritas en los diferentes espacios regionales.

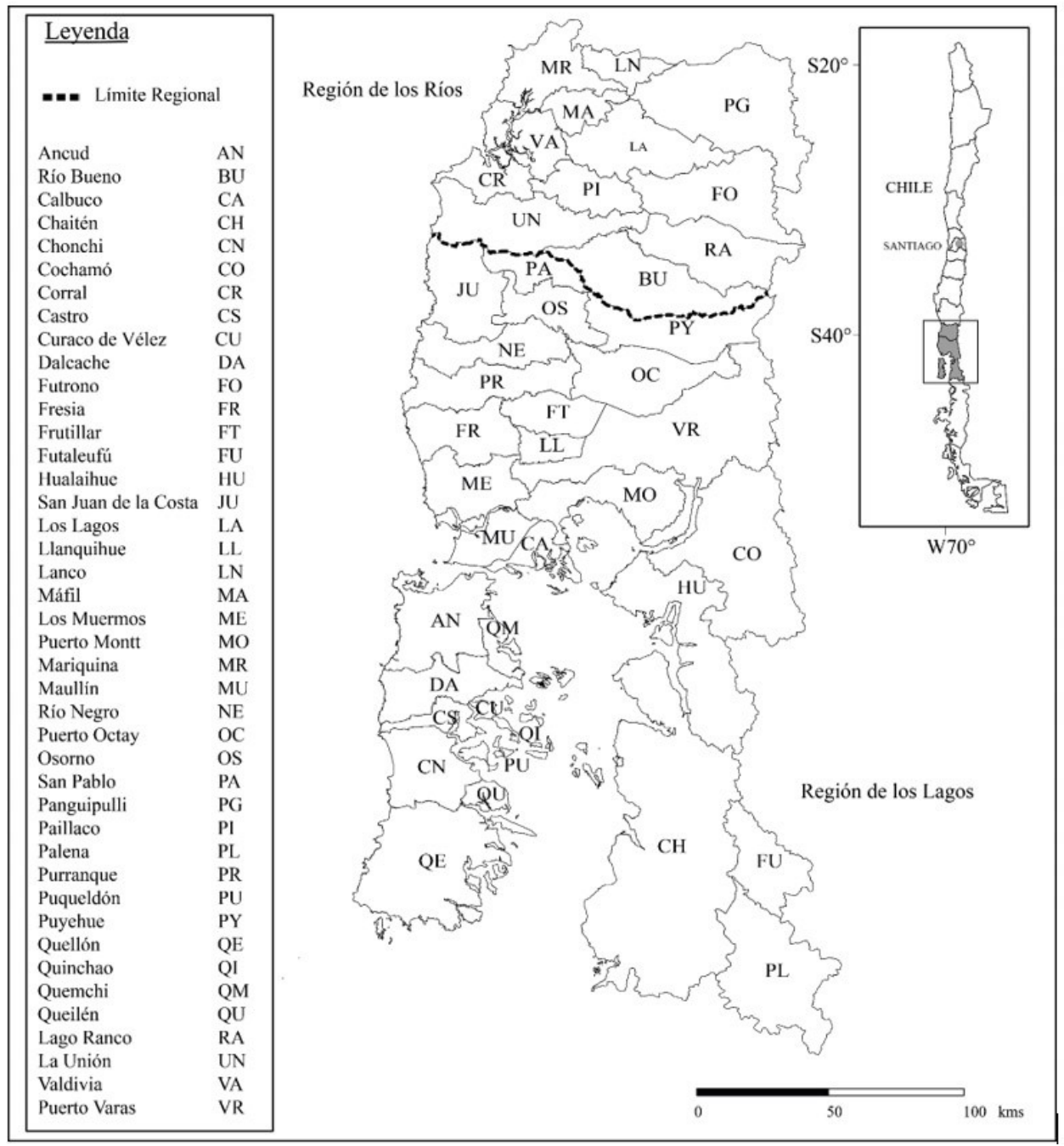

Figura 1. Comunas de las regiones de Los Ríos y Los Lagos. Fuente: Elaboración propia a partir de archivo shape file descargado del sitio web: http://www.bcn.cl (2018)

Figure 1. Communes of the Los Ríos and Los Lagos regions. Source: Own elaboration based on shape file http://www.bcn.cl (2018)

\section{Material y métodos}

El diseño metodológico de la presente investigación descansa en tres pasos. El primero la recolección de datos, un segundo en el cual se elaboran las matrices y finalmente la aplicación del modelo.

En un primer momento, se identificaron las comunas pertenecientes a las Regiones de 
Los Ríos y Los Lagos. Se contabilizaron un total de 42 comunas distribuidas disparmente en el territorio, de las cuales $32(76 \%)$ pertenecen administrativamente a la Región de Los Lagos y sólo 10 equivalentes al $24 \%$ a la de Los Ríos (figura 1).

Para el análisis se establecieron cuatro periodos o intervalos de años. Estos correspondieron a 1977-1982, 1987-1992, 1997-2002 y 2007-2012. Su selección se justifica por la disponibilidad de datos provenientes de los diferentes censos de población en Chile y la pregunta en tales censos referente a la migración interna ¿En qué comuna o país vivía usted hace cinco años? Además, los periodos de tiempo seleccionados tienen relación a poder testear durante cuatro periodos el modelo y considerar un momento antes y posterior del proceso de segmentación del espacio regional.

Considerando lo anteriormente expuesto, se procedió a la obtención de los datos. Para los tres primeros periodos, se accedió al sitio online de CELADE más específicamente de la Base de Datos de Migración Interna en América Latina y el Caribe, obteniendo tres matrices (una por cada periodo) de los censos de 1982, 1992 y 2002 que fueron proporcionados a tal institución por el Gobierno de Chile. Para el periodo 20072012 se extrajo la información desde el censo (no oficial) del año 2012 a través del programa REDATAM. De tal forma, se obtuvieron 4 matrices de tamaño $42 \times 42$ para cada año, que incorporan las comunas correspondientes. Se modificó el nombre de todas las comunas a una clave de sólo 2 letras únicas e irrepetibles (figura 1) para facilitar la lectura de las cartas. Finalmente se realizó una matriz con la población total de cada comuna para cada año en específico.
En un tercer momento se empleó el modelo gravitatorio mediante el software libre $\mathrm{y}$ gratuito R (con su interfaz Rstudio), considerando la utilización de los paquetes reshape2, sp y maptools, además de dos archivos vectoriales procesados en Qgis (versión 2.18). El tipo de modelo aplicado correspondió al modelo gravitatorio tipo Wilson de doble restricción origen y destino (ecuación 4$)^{2}$.

$$
T i j=A i \text { Oi Bj Dj }\left(e^{-\beta c i j}\right)(4)
$$

El modelo tipo Wilson de doble restricción, indica que Tij corresponde al flujo total entre i y j. Ai y Bj son parámetros de conservación (doble restricción). Oi y Dj son las masas (población) y cij es el costo de desplazarse entre i y j (distancia), la cual, en este caso, funciona como constante.

Una vez que se aplicó el modelo y tal como se indicó en el primer apartado, se calcularon los efectos barreras y preferenciales a partir de la resta entre el valor real del flujo y el modelado. Como la resta entre tales valores genera una nueva matriz, se procedió a normalizar los valores residuales obtenidos y calcular la desviación estándar, valores máximos, mínimos y los tres primeros cuartiles para establecer un umbral y así generar las cartografías de flujos para cada periodo.

Por último, cabe destacar que la selección del tal tipo de modelo, obedece a las sugerencias planteadas por Parrochia (2006) y Pumain \& Saint-Julien (2001) y a los resultados obtenidos por Maturana et al. (2016). Tales autores, señalan que, dentro de este tipo de modelos, el tipo Wilson es el que presentó un mejor ajuste en los testeos efectuados, alcanzado un R2 de 73,7\%. Como se aprecia en los siguientes párrafos, para este caso se logró incluso un mejor ajuste.

1 Los datos obtenidos del Censo año 2012 son de carácter referencial, los cuales permiten una aproximación a los flujos migrantes de tipo interno.

2 Cabe señalar, que, para la programación del modelo se adaptó el script elaborado en R por Claude Grasland y Laurent Beauguitte. Se agradece por parte de los autores, el aporte. 


\section{Resultados y discusión}

Tras la aplicación del modelo y antes de su interpretación, es relevante y útil caracterizar algunos resultados de su aplicación en relación a los grados de ajuste. Así, se estimó el coeficiente de determinación (R2) para determinar el porcentaje de fiabilidad que entrega dicho modelo al estimar los flujos de migración interna.

En la tabla 2 se observa la calidad de ajuste del modelo para el primer intervalo de años (1977-1982), la cual es de un $67,8 \%$. Se destaca que, al aumentar el año, el R2 mejora de manera considerable, alcanzado casi un $90 \%$ para el último periodo (2007-2012). Lo anterior podría ser explicado por diversos motivos tales como mejor calidad de los datos, mayor universo muestreado, etc. En efecto, Maturana et al. (2016) experimentaron la misma situación. Además, se elaboraron cuatro gráficos para observar el grado de ajuste entre los flujos modelados (eje y) respecto a los observados (eje x) (figura 2).

\section{Tabla 2}

Coeficiente de determinación (R2) obtenido de los intervalos de años 1977-1982, 1987-1992, 1997-2002 y 2007-2012. Fuente: Elaboración propia (2018)

Table 2

Coefficient of determination (R2) obtained from the intervals of years 1977-1982, 1987-1992, 1997-2002 and 2007-2012. Source: Own elaboration (2018)

\begin{tabular}{|c|c|c|c|c|c|c|c|c|c|c|c|}
\hline \multirow{2}{*}{$\begin{array}{l}\text { Intervalos } \\
\text { de Años }\end{array}$} & \multirow{2}{*}{$\mathrm{R} 2$} & \multicolumn{2}{|c|}{$\begin{array}{l}\text { Desviación } \\
\text { estándar }\end{array}$} & \multicolumn{2}{|c|}{ Máximo } & \multicolumn{2}{|c|}{ 1er cuartil } & \multicolumn{2}{|c|}{ Mediana } & \multicolumn{2}{|c|}{ 3er cuartil } \\
\hline & & FR & FE & FR & FE & FR & $\mathrm{FE}$ & FR & $\mathrm{FE}$ & FR & FE \\
\hline $1977-1982$ & 67,8 & 155,4 & 105,4 & 2.300 & $1.250,3$ & 3 & 2 & 10 & 6 & 40 & 24 \\
\hline 1987-1992 & 78,6 & 114,9 & 53,8 & 991 & 181 & 3 & 8 & 10 & 11 & 38 & 22 \\
\hline $1997-2002$ & 81,4 & 137,7 & 138,8 & 1.785 & 472 & 4 & 1 & 11 & 6 & 41 & 32 \\
\hline $2007-2012$ & 89,0 & 191,8 & 59,2 & 5.543 & 220 & 3 & 3 & 9 & 4 & 29 & 46 \\
\hline
\end{tabular}

FR: Flujo real. FE: Flujo estimado o modelado.

Los puntos encontrados bajo la recta son subestimados por el modelo, vale decir el modelo calcula un número inferior de personas migrantes de las que se esperaría en función de la interacción que están presentados los territorios. En cambio, los puntos ubicados sobre la recta significan una sobrestimación del modelo, conduciendo a una mayor asignación de flujos en función de lo esperable.

Al generar gráficos de ajuste e histogramas para el flujo residual (flujo observado-flujo modelado) de cada periodo de tiempo se obtiene información relevante como por ejemplo la localización de los datos y la caracterización de potenciales efectos preferenciales/barreras (figura 2).

Concretamentesielflujoresidualespositivo es sinónimo de efectos preferenciales entre las comunas, en cambio sí es negativo los flujos migratorios entre adquieren características de barrera, es decir, entre ambos territorios hay fricciones para la interacción según el modelo. 
a)

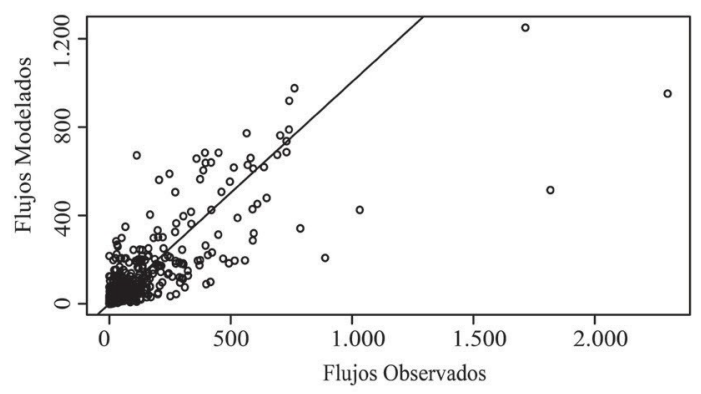

c)

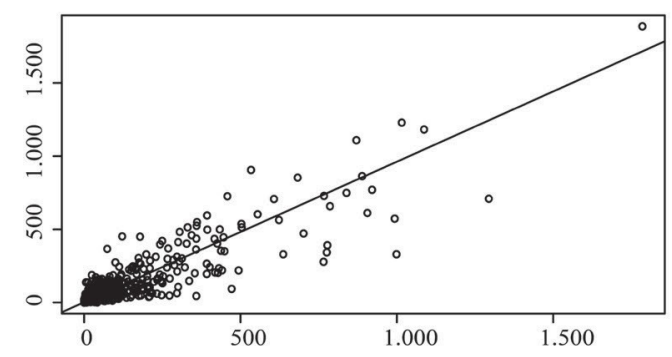

b)

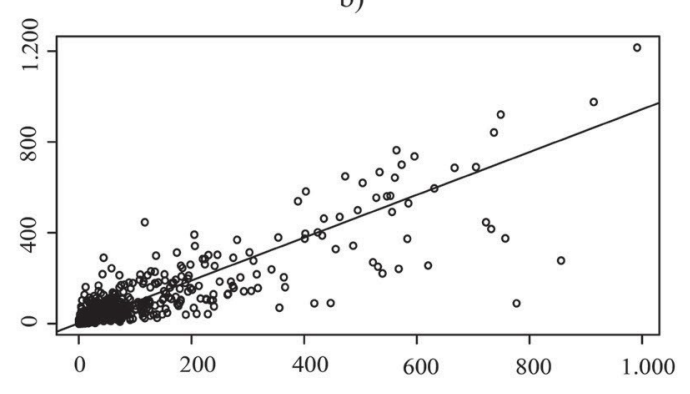

d)

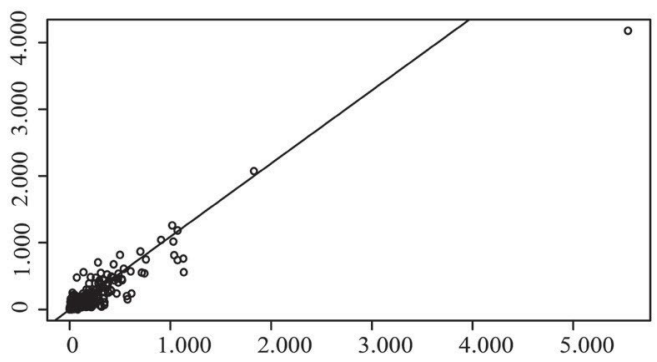

Figura 2. Ajuste entre flujos observados y estimados para los intervalos de años 1977-1982 (a), 1987-992 (b), 1997-2002 (c) y 2007-2012 (d). Fuente: Elaboración propia (2018)

Figure 2. Adjustment of data observed and estimated flows for the intervals of years 1977-1982 (a), 1987-992 (b), 1997-2002 (c) and 2007-2012 (d). Source: Own elaboration (2018)

En este sentido, se estimó de utilidad caracterizar los valores residuales obtenidos para los diferentes periodos. Para el 1977-1982 (figura 2a y figura 3) existe una importante amplitud de los datos, donde el mínimo supera -500 y el máximo flujo residual se acerca a 1.500 (figura 3). Sin embargo, se debe destacar que un porcentaje importante de los residuales obtenidos, $(70 \%)$ son de muy baja intensidad ya que se ubican en valores entre -13 y 7.

Entre los años 1987-1992 (figura 2b y figura 3), es posible apreciar la misma lógica del anterior: amplia diferencia entre el máximo y menor flujo, baja intensidad (tanto positiva como negativa) de la mayor parte de los datos ( $70 \%$ aproximadamente).
Para el siguiente periodo (19972002) (figura 2c y figura 3 ) se repite la concentración de la mayor parte de los datos en flujos de baja intensidad, sin embargo, la amplitud entre el máximo y mínimo disminuye de 2.500 en 1977-1988 a 1.400 para los años comprendidos entre 1997 y 2002 (figura 3), significando una relativa homogenización de los efectos barrera/preferenciales que se manifiestan entre las comunas del área de estudio, deduciéndose un sistema de comunas estables que tienden a retener a gran parte de su población (figura 1).

Por último, entre los años 2007 y 2012 (figura 2 y figura 3 ) nuevamente los datos se concentran en la parte media del grafico señalizando flujos residuales débiles entre 
las comunas. Al analizar el grafico de dispersión para este mismo periodo llama la atención que la diferencia entre valor máximo y mínimo se reduce a tan solo 1.000, una clara reducción en comparación con periodos anteriores, no obstante, hay un dato que se escapa (en forma positiva) en relación al resto de datos, expresando un claro efecto migratorio preferente.

En las figuras 4 y 5 se observan las cartografías de los de los flujos preferenciales y barrera estimados por el modelo. A continuación, se explican dichas figuras para cada periodo correspondientemente:

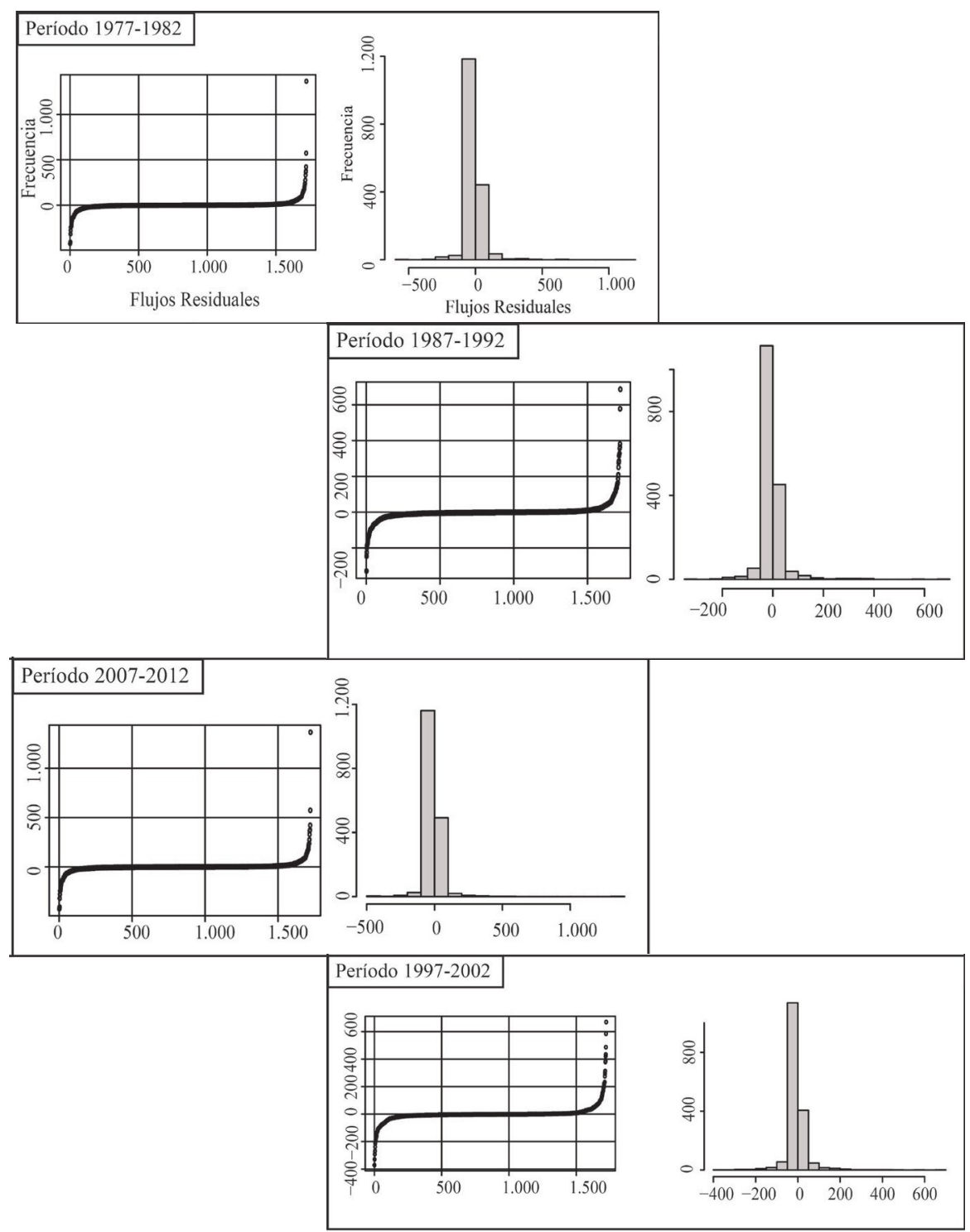

Figura 3. Valores residuales expresados en gráficos de dispersión e histogramas para los intervalos de años 1977-1982, 1987-992, 1997-2002 y 2007-2012. Fuente: Elaboración propia (2018)

Figure 3. Residual values in scatter plots and histograms for the intervals of years 1977-1982, 1987-992, 1997-2002 and 2007-2012. Source: Own elaboration (2018) 

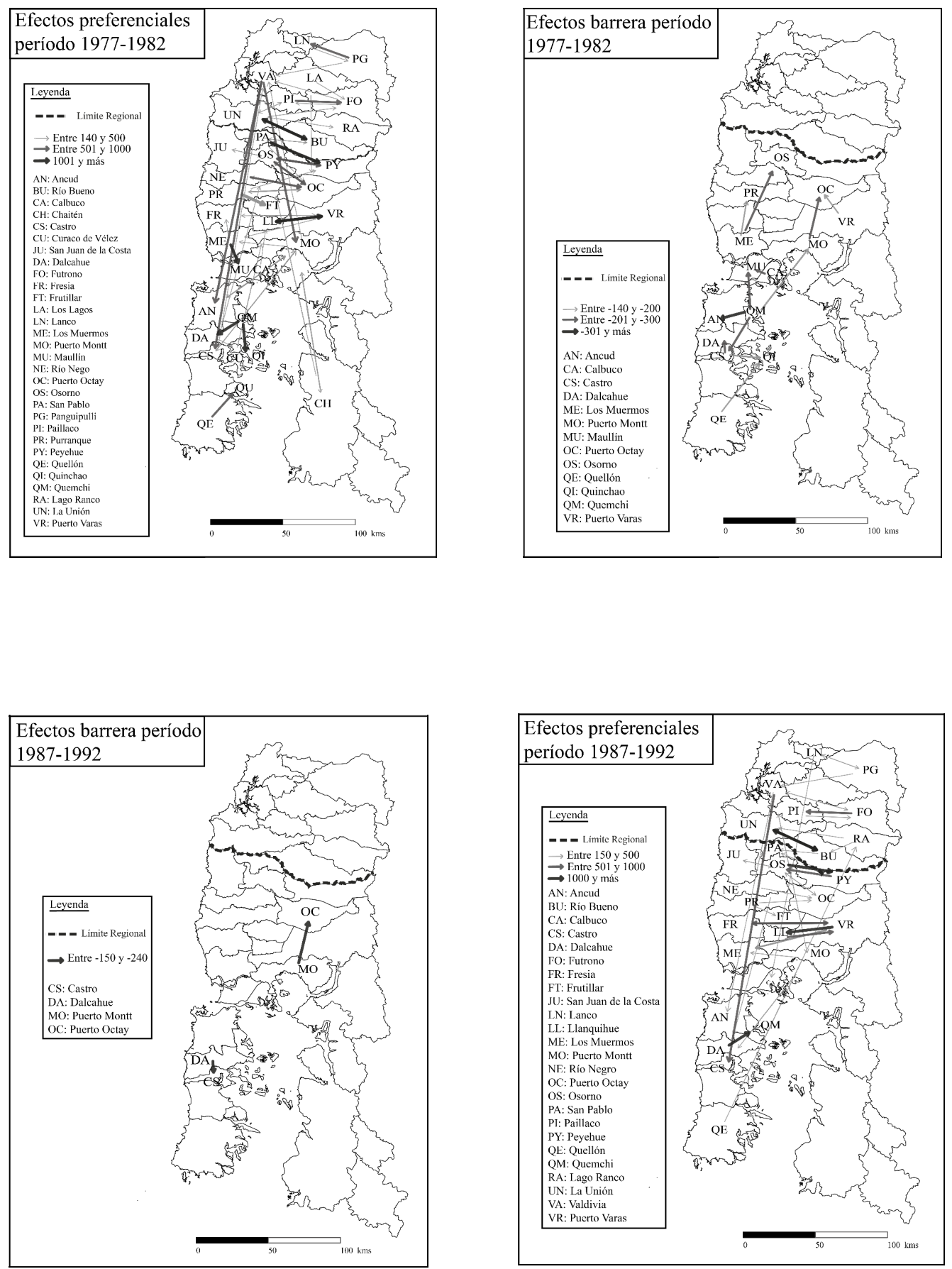

Figura 4. Efectos preferenciales y barrera periodos 1977-1982 y 1987-1992. Fuente: Elaboración propia a partir de INE 1982 y 1992.

Figure 4. Preferential effects and barrier periods 1977-1982 and 1987-1992. Source: Own elaboration based on INE 1982 and 1992. 

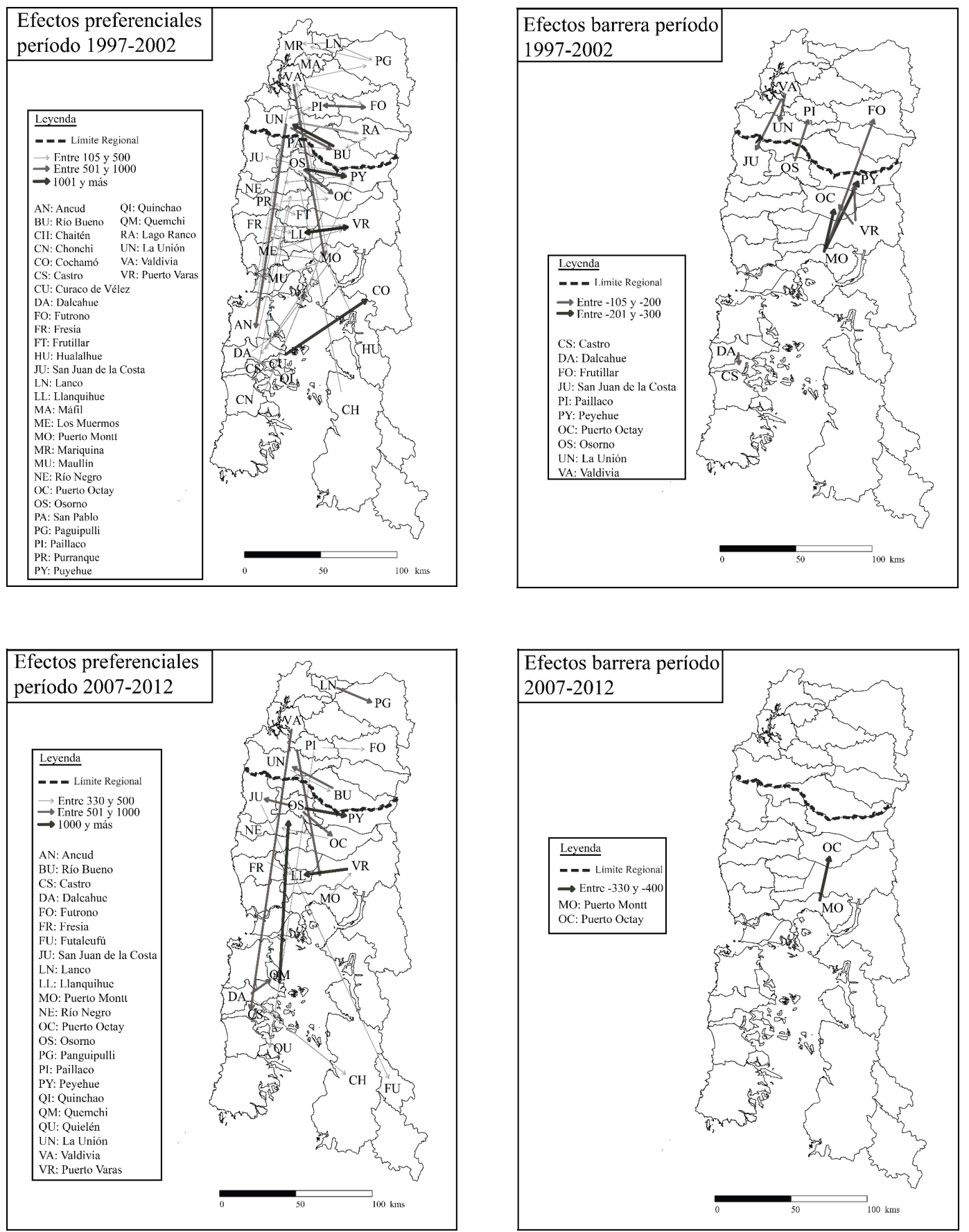

Figura 5. Efectos preferenciales y barrera periodos 1997-2002 y 2007-2012. Fuente: Elaboración propia a partir de INE 2002 y 2012.

Figure 5. Preferential effects and barrier periods 1997-2002 and 2007-2012. Source: Own elaboration based on INE 2002 and 2012. 
Para el primer periodo de tiempo (19771982) en cuanto efectos preferenciales se visualiza una fuerte preferencia en la Isla de Chiloé, más específicamente de los flujos migrantes de Quemchi en dirección de Dalcachue (+2.240) y Quinchao (+3.297), es importante señalar que dichas comunas son principalmente de carácter rural, sin embargo, el efecto preferencial en este caso se puede explicar con el auge de la industria salmonera desarrollada en los últimos años en tales territorios (Barton, 1997; Román et al., 2016).

Otro aspecto significativo a señalar es la preferencia migratoria (en ambas direcciones) entre comunas ubicadas en la costa y el valle central (no incluyendo la isla de Chiloé) dando cuenta de una fuerte dinámica de flujos de personas entre dichas zonas, siendo principalmente llamativo el generado entre Llanquihue y Puerto Varas $(+1356$ y +1235), ambas comunas con un vasto desarrollo turístico.

En cuanto a efectos barreras en este intervalo de tiempo destacan dos casos en particular. El primero, las comunas de Puerto Montt y Puerto Varas en las cuales la población presenta resistencia de migrar hacia Puerto Octay (-229 y -168 respectivamente). Otro caso es lo observado en la Comuna de Quemchi, cuyos habitantes no buscan migrar hacia las comunas de Ancud (-465), Castro (-229), Los Muermos (-216) y Puerto Montt (-194), siendo especialmente altacon la primera comuna respectivamente. Tales características podrían atribuirse a ciertos movimientos pendulares en desmedro de los migratorios, sin embargo, considerando las posibilidades de movilidad algo más limitadas en transporte para tal periodo (entre 1977-1982), su impacto en este caso debería seracotado.

Al igual que el periodo anterior, los flujos migratorios en el periodo 1987-1992 tienden a preferir intercambios entre comunas del interior y costera en ambos sentidos, destacando principalmente la relación $\mathrm{La}$ Unión y Río Bueno en la Región de Los Ríos (+1.398). Tales aspectos van en la línea con las teorías de interacción espacial, que, a pesar de todas las mejoras y disminución de costos en transporte y telecomunicaciones, determinados flujos disminuyen a medida que la distancia aumenta o se circunscriben a espacios que históricamente han sido definidos (un caso sería en la División Político Administrativa DPA). Por ejemplo, en el caso inglés, se observa muy bien como finalmente la interacción espacial de las personas, sigue en línea con los límites políticos administrativos del territorio (Ratti et al., 2010).

A su vez en la Región de Los Lagos destacan efectos preferenciales observados entre Osorno con Puyehue $(+1.199$ y $+566)$, Llanquihue y Puerto Varas $(+1.164$ y +824) y Fresia-Puerto Varas $(+629$ y +255 ), siendo el común denominador de la mayoría de estas comunas estar ubicadas en la zona lacustre, zona turística por excelencia (Soza-Amigo, Rosales, \& Aroca, 2016) y presentando una proximidad en términos espaciales. Sin duda es relevante mencionar que la preferencia migratoria más importante que existe para este periodo es la Dalcahue a Quemchi (+5.273), situadas solamente a 51 kilómetros de distancia y cuyo valor va en sentido contrario al que se mostraba para el intervalo anterior.

Respecto a los efectos barrera, estos son pocos numerosos y bajos en intensidad para este periodo, destacando la resistencia de migrar desde Puerto Montt a Puerto Octay (-208), característica que se mantiene a través del tiempo, dado que ya se encontraba presente entre 1977 y 1982, a esto se suma otra reticencia migratoria desde la comuna de Dalcahue a Castro (-243). 
Entre 1997 y 2002 los flujos preferenciales presentan diferencias sustanciales cuando son comparados con los periodos anteriores, producto seguramente de las transformaciones que en tal periodo se intensifican en la industria del salmón (Bustos, 2012). Principalmente se observan mayores preferencias migratorias interregionales: Ancud-Valdivia (+198), Puerto Montt-Valdivia (+550), La UniónAncud (+770) siendo esta última la de mayor intensidad. Es decir, se está en presencia de algunas migraciones entre espacios cuya distancia geográfica es mayor. Las preferencias costa-interior siguen desarrollándose, aunque pierden intensidad en desmedro de los flujos preferenciales al interior-zonas lacustres, destacando la importante intensidad que muestra la relación Llanquihue-Puerto Varas (+2.187 y +1.076). La situación en la Isla de Chiloé se mantiene similar a años anteriores, exceptuando por una relevante preferencia migratoria de la Comuna Curaco de Vélez hacia Cochamó (+1.437) y donde es posible apreciar nuevamente una cercanía en términos geográficos.

Los efectos barrera se multiplican para este periodo llamando la atención tres elementos centrales: en primer lugar, la resistencia a migrar desde la capital regional Puerto Montt hacia comunas ubicadas más al norte tales como Puerto Octay (-240) que se repite nuevamente y Puyehue (-233). Además, se observa una barrera migratoria desde Puerto Varas hacia las mismas comunas nombradas anteriormente (-140 y -112 respectivamente) y por último un notorio efecto barrera interregional (interprovincial en ese tiempo) entre las comunas de Valdivia-San Juan de la Costa (-126), Osorno-Paillaco (-111) y Puerto Montt-Futrono (-105).
En este caso, para al menos la Región de Los Lagos, tales efectos barrera podrían vincularse a procesos de conmutación, seguramente por las mejores en transporte y comunicaciones, que permitían una mayor interacción sin la necesidad de migrar. Además, habría que agregar las dinámicas de las ciudades de Puerto Montt y Puerto Varas (Escolano \& Ortiz, 2004), que seguramente inciden en que la población desee restar en tales espacios.

El análisis del periodo (2007-2012) resulta relevante ya que en el año 2007 es creada la nueva Región de los Ríos, a partir de la ex provincia de Valdivia que pertenecía a la Región de Los Lagos. En tal contexto, se aprecia una fuerte e intensa preferencia migratoria desde Quemchi a Osorno $(+1.407)$. Tal valor residual es el más elevado, el resto de efectos preferenciales son de baja intensidad comparados con el mayor anteriormente señalado, existiendo dos flujos interregionales: desde la comuna de Valdivia hacia Castro $(+520)$ y PaillacoPuerto Varas (+432). Por último, se constata un efecto barrera para este intervalo desde la comuna de Puerto Montt hacia Puerto Octay (-347), esta resistencia se ha repetido en los cuatro periodos de tiempo analizados.

Así, si se analiza de manera particular la matriz de valores residuales para los últimos dos periodos en cuestión, referente a los 20 residuales que expresan preferencia migratoria y los 20 más destacables efectos barrera, se pudo constatar que para ambos periodos la mayoría de las preferencias migratorias se circunscriben a sus espacios regionales (tabla 3), por otro lado, la proporción de los valores residuales inter regionales se aprecian muy bajos, alcanzando para el periodo 1997-2012 un 15\%. 
Tabla 3

Los 40 valores residuales más relevantes, respecto a su origen intra regional o inter regional.

Fuente: Elaboración propia (2018)

Table 3

The 40 most relevant residual values, with respect to their intra-regional or inter-regional origin. Source: Own elaboration (2018)

\begin{tabular}{lll}
\hline Preferencias migratorias & & \\
Tipo/periodo & $1997-2012$ & $1997-2002$ \\
Intra regional & $85 \%$ & $80 \%$ \\
Inter regional & $15 \% \mathrm{~h}$ & $20 \%$ \\
\hline Efectos barrera & & \\
Tipo/periodo & $1997-2012$ & $1997-2002$ \\
Intra regional & $70 \%$ & $60 \%$ \\
Inter regional & $30 \%$ & $40 \%$ \\
\hline
\end{tabular}

Por otro lado, al analizar los efectos barrera, se aprecia que (para el periodo 1997-2012) los residuales intra regionales representan también los mayores tipos de migración. Lo anterior, indicaría que hay ciertas comunas donde sus habitantes se resisten a migrar intra regionalmente, aspecto que sería esperable, particularmente cuando se observa que un importante porcentaje de los casos analizados evidencia que tales espacios corresponden a Puerto Montt o Valdivia (60\% de los casos analizados), es decir, los habitantes de tales territorios prefieren continuar residiendo en tales comunas, en vez de migrar a otras.

No obstante, al analizar el periodo 19972002, la situación aparecía matizada, es decir, toman relevancia las resistencias a las migraciones inter regionales y una relativización de las intra regionales (tabla 3).

A partir de lo anterior y según las hipótesis planteadas al comienzo de este trabajo, es que no se evidencian patrones claros que demuestren un mayor proceso migratorio entre las regiones de Los Ríos y Los Lagos, pero lo interesante, es que incluso tal situación se evidencia de manera anterior al proceso de segmentación del espacio, donde inclusive ya existía una mayor resistencia a migrar inter regionalmente (en ese momento particular entre provincias). Esto podría indicar que efectivamente la región de Los Ríos, al menos en términos migratorios, se conformaba como una estructura homogénea, diferenciada de la Región de Los Lagos, antes de su creación.

\section{Conclusiones}

Sería posible caracterizar desde dos aspectos las conclusiones en este trabajo. Por una parte, la aplicación del modelo y por otra, los procesos migratorios.

Respecto a las primeras, la utilidad y eficacia del modelo gravitatorio aplicado al estudio de las migraciones internas, más específicamente en las regiones de los Lagos y de Los Ríos es confiable, quedando demostrado en los valores de $\mathrm{R}^{2}$ que se obtuvo del modelo para los distintos 
periodos analizados, logrando casi un $90 \%$ de explicación del juego de datos. Esto entrega una confiabilidad para posibles aplicaciones de tal modelo al estudio de las migraciones interna, como por ejemplo en instrumentos de planificación territorial, principalmente si pensamos en la componente urbana de los nuevos Planes Regionales de Ordenamiento Territorial (PROT), lo anterior considerando su rápida aplicación y sencillez en los datos necesarios.

No obstante, se debe hacer hincapié que se trata de un modelo y por tanto una simplificación de la realidad. En efecto, existen múltiples variables territoriales que no son incorporadas, por tanto, se deben considerar tales limitantes al momento de su interpretación. Así, sería interesante testear modelos de radiación para próximos trabajos.

En un segundo momento y en referencia a los resultados obtenidos para las migraciones del área de estudio, es posible concluir que los procesos migratorios se han circunscritos más bien a los respectivos espacios regionales, incluso antes del proceso de segmentación del espacio donde se crea la Región de Los Ríos, lo cual en cierta manera podría reforzar la idea que tal territorio se constituye como una unidad funcional diferente a Los Lagos, más allá de las relaciones propias entre territorios.

Sumando a lo anterior y quizás de manera esperable, existe una creciente resistencia a migrar de las comunas con mayor población hacia otras de menor cantidad de habitantes, principalmente desde las capitales regionales como Valdivia, Puerto Montt u otras como Osorno y Puerto Varas, lo cual refuerza las ideas de estudiar los desafíos que imponen los procesos de conmutación o movimientos pendulares que podrían ser cada vez más significativos. En este sentido, futuras investigaciones podrían incorporar estas ideas y explorar tales procesos.

\section{Agradecimientos}

Proyecto Fondecyt Iniciación 11150087 financiado por el Gobierno de Chile, para elaborar este trabajo.

\section{Referencias}

Barton, J. \& Román, A. (2016). Sustainable development? Salmon aquaculture and late modernity in the archipelago of Chiloé, Chile. Island Studies Journal, 11(2), 651672. Recuperado de https://www. islandstudies.ca/sites/islandstudies.ca/ files/ISJ-11-2-MS369-Barton+Roman. pdf.

Barton, J. (1997). ¿Revolución azul? El impacto regional de la acuicultura del salmón en Chile. Revista EURE, 23(68), 57-76. Recuperado de http://www.eure. cl/index.php/eure/article/view/1156

Batty, M. (2013). The new science of cities. Massachusetts: Institute Technology (MIT). ISBN: 9780262019521

Busso, G. (2014). Argentina, Bolivia, Brasil y Chile: pobreza y efectos sociodemográficos de la migración interna a inicios del siglo XXI. Notas de Población, (84), 53-85. Recuperado de https://repositorio.cepal.org//handle/11 $362 / 12815$ 
Bustos, B. (2012). Brote del virus ISA: crisis ambiental y capacidad de la institucionalidad ambiental para manejar el conflicto. Revista EURE, 38(115), 219245. https://dx.doi.org/10.4067/S0250-71 612012000300010

CELADE (s.f). Base de datos de Migración Interna en América Latina y el Caribe (MIALC). Santiago de Chile: Banco de datos CELADE. Recuperado de https://www.cepal.org/celade/migracion/ migracion_interna/

Chen, Y. \& Wang, F. (2008). Fourier analysis of an expanded gravity model for spatio-temporal interactions. Far East Journal of Dynamical Systems, 10(3), 325347. Recuperado de https://arxiv. org/ftp/arxiv/papers/1306/1306.3820.pdf

Commenges, H. (2016). Modèle de radiation et modèle gravitaire : du formalisme à l'usage. Revue Internationale de Géomatique, 26(1), 79-95. https://doi. org/10.3166/RIG.26.79-95

Di Filippo, A. \& Bravo, R. (1977). Los centros nacionales de desarrollo y las migraciones internas en América Latina: un estudio de casos, Chile. Revista EURE, 5(14), 67-101. Recuperado de http://www. eure.cl/index.php/eure/article/view/897

Duffus, L., Alfa A., \& Soliman, A. (1987). The reliability of using the gravity model for forecasting trip distribution. Transportation, 14(3), 175-192. https:// doi.org/10.1007/BF00837528

Eichenbaum, J. (1975). A Matrix of Human Movement. International
Migration, 13(1-2), 21-41. https://doi. org/10.1111/j.1468-2435.1975.tb00012.x

Escolano, S. \& Ortiz, J. (2004). La complejidad de los procesos de reestructuración socio espacial de las ciudades intermedias. Anales de geografía de la Universidad Complutense, 24, 79-106.

Faura, U. \& Gómez, J. (2001). Modelos migratorios: una revisión. Revista Asturiana de economía, 21, 209-235.

Fløysand, A., Barton, J., \& Román, Á. (2010). La doble jerarquía del desarrollo económico y gobierno local en Chile: El caso de la salmonicultura y los municipios chilotes. Revista EURE, 36(108), 123-

148. Recuperado de http://www.eure.cl/ index.php/eure/issue/view/131

Haynes, K. \& Fotheringham, A. (1984). Gravity and spatial interaction models. Beverly Hills, CA: Sage Publication. ISBN: 0803923260

Instituto Nacional de Estadísticas de Chile (INE) (2007). Migraciones regionales 1992-2002. Documento de trabajo, Departamento de Demografía, Geografía y Censos, Gobierno de Chile, Santiago: Gobierno de Chile

Instituto Nacional de Estadísticas de Chile (INE) (2012). Censo nacional de población año 2012. Base de datos, Santiago: Gobierno de Chile.

Instituto Nacional de Estadísticas de Chile (INE) (2017). Resultados definitivos Censo 2017. Santiago: Gobierno de Chile. 
Kang C., Liu, Y., Guo, D., \& Qin, K. (2015) A generalized radiation model for human mobility: spatial scale, searching direction and trip Constraint. Plus one, 10(11), e0143500. https://doi. org/10.1371/journal.pone. 0143500

Karamera, D., Iwuagwu, V., \& Davis, B. (2000). A gravity model analysis of international migration to North America. Applied Economics, 32(13), 1745-1755. https:// doi.org/ 10.1080/ 000368400 421093

Levy, J. \& Lussault, M. (2013). Dictionnaire de géographie et de l'espace des sociétés. France: Belin Litterature et revues. ISBN: 978-2701163956.

Maturana, F., Rojas, A., \& Poblete, D. (2016). Análisis y tendencias migratorias en la región del Biobío (Chile) entre 1982 y 2002. Aplicación desde el modelo gravitacional. Revista Economía, Sociedad y Territorio, 16 (52), 729-759. https://doi.org/10.22136/est0522016638

Martínez, J. (2002). Ciudades de Chile, Migración Interna y Redistribución de la Población: Algunas Evidencias del Periodo 1987-1992. Revista de geografia Norte Grande, 29, 21-38. Recuperado de https://repositorio.uc.cl/ handle/11534/10441

Maturana, F. \& Rojas, A. (Eds.) (2015). Ciudades intermedias en Chile: Territorios olvidados. Santiago: Ril Editores. ISBN: 978-956-01-0195-2

Ortiz, J. \& Morales, S. (2002). Impacto socioespacial de las migraciones intraurbanas en entidades de centro y de nuevas periferias del Gran Santiago. Revista EURE (Santiago), 28(85), 171-185. https://dx.doi.org/10.4067/S0250-7161 2002008500009

Parrochia, D. (2006). Pour une théorie de la relativité géographique (Vers une généralisation du modèle gravitaire). Cybergeo, 337. https://doi.org/10.4000/ cybergeo. 2407

Piras, R. (2016). A long-run analysis of push and pull factors of internal migration in Italy. Estimation of a gravity model with human capital using homogeneous and heterogeneous approaches. Papers in Regional Science, 96(3), 571-602. https://doi.org/10.1111/pirs.12211

Pumain, D. \& Saint-Julien, T. (2001). Análisis espacial. Las interacciones. Santiago: Pontificia Universidad Católica de Chile y Universidad de Concepción. Serie GEOlibros, 21. ISBN 9789561414662

Ratti C., Sobolevsky S., Calabrese F., Andris C., Reades J., Martino M., ... Strogatz, S. (2010). Redrawing the Map of Great Britain from a Network of Human Interactions. Plus one, 5(12), e14248. https://doi.org/10.1371/journal. pone. 0014248

Ravenstein, E. (1885). The Laws of Migration. Journal of the Statistical Society, 48(2), 167-235.

Ravenstein, E. (1889). The Laws of Migration - Second Paper. Journal of the Royal Statistical Society, 52(2), 241-305. 
Rodrigo, L. \& Atienza, M. (2014). Migración y representaciones regionales: discursos sobre la Región de Antofagasta. Revista EURE, 40(120), 159-181. https://doi.org/10.4067/s025071612014000200008

Rodríguez, J. (2004). Migración interna en América Latina y el Caribe: estudio regional del periodo 1980-2000. Santiago: CEPAL. ISBN: 92132233548

Rodríguez, J. \& González. G. (2006). Redistribución de la población y migración interna en Chile: continuidad y cambio según los últimos cuatro censos nacionales de población y vivienda. Revista de geografía Norte Grande, 35, 7-28. http://dx.doi.org/10.4067/S071834022006000100002

Román, A., Barton, A., Bustos B., \& Salazar, A. (Eds.). (2016). Revolución salmonera. Paradojas y transformaciones territoriales en Chiloé. Santiago: RIL Editores. ISBN: 978-956-01-0256-0

Roy, J. (2004). Spatial Interaction modelling: a regional science context. Berlin: Springer. https://doi. org/10.1007/978-3-540-24807-1

Schiappacasse, P., Contreras, M., \& Fuensalida, C. (2001). Migraciones internas hacia la Región Metropolitana de Santiago de Chile: una comparación con planteamientos teóricos. Investigaciones Geográficas, 35, 1-25. https://doi. org/10.5354/0719-5370.2001.27735

Simini, F., González, M., Maritan, A., \& Barabási, A. (2012). A universal model for mobility and migration patterns. Nature, 484(7392), 96-100. https://doi. org/10.1038/nature10856

Stefanouli, M. \& Polyzosa, S. (2017). Gravity vs radiation model: two approaches on commuting in Greece. Transportation research procedia, 24, 65-72. https://doi.org/10.1016/j. trpro.2017.05.069

Soza-Amigo, S., Rosales, C., \& Aroca, P. (2016). Análisis de los centros primados vinculados al turismo en el Parque Nacional Puyehue. Revista de geografia Norte Grande, 65, 179-195. https://doi. org/10.4067/s0718-34022016000300009

Stewart, J. Q. (1941). An inverse distance variation for certain social influences. Science, 93(2.404), 89-90. https://doi. org/10.1126/science.93.2404.89

Stewart, J. Q. (1948). Demographic gravitation: evidence and applications. Sociometry, 11 (1/2), 31-58. https://doi. org $/ 10.2307 / 2785468$

Thomas, R. \& Huggett, R. (1980). Modelling in geography: a mathematical approach. London: Harper \& Row. ISBN: 0389200492

Vial, C., Maturana, F., \& Rojas, A. (2016). Nuevas regiones e inversión pública en Chile: El caso de las regiones de Los Ríos y Arica y Parinacota. GIGAPP Estudios Working Papers, 2016-12, 1-20. ISSN: 2174-9515

Wilson, A. (1971). A family of spatial interaction models and associated 
developments. Environment and Zubelzu, S., López, A., Gutiérrez, M., Planning A: Economy and Space, 3(1), 1- \& Blanco, F. (2011). Los métodos 32. https://doi.org/10.1068/a030001 gravitacionales como herramienta para el cálculo de las emisiones de gases de efecto

Zelinsky, W. (1971). The hypothesis of the invernadero derivadas del tráfico rodado en mobility transition. Geographical Review, 61(2), 219-249. https://doi. la planificación urbana. Revista ingeniería org/10.2307/213996 de construcción, 26(2), 187-207.https://doi. org/10.4067/s0718-50732011000200004 